Article

\title{
Clinical Relevance of Antibiotic Susceptibility Profiles for Screening Gram-negative Microorganisms Resistant to Beta-Lactam Antibiotics
}

\author{
Francisco Montiel-Riquelme ${ }^{1}{ }^{10}$, Elisabeth Calatrava-Hernández ${ }^{2}$, Miguel Gutiérrez-Soto ${ }^{3}$, \\ Manuela Expósito-Ruiz ${ }^{4}\left[\right.$, José María Navarro-Marí ${ }^{2}$ and José Gutiérrez-Fernández ${ }^{1,2, *} \mathbb{C}$ \\ 1 Department of Microbiology, School of Medicine, University of Granada-ibs, 18012 Granada, Spain; \\ fmontiel@correo.ugr.es \\ 2 Department of Microbiology, Hospital Universitario Virgen de las Nieves-ibs, 18012 Granada, Spain; \\ elisabeth.calatrava.sspa@juntadeandalucia.es (E.C.-H.); josem.navarro.sspa@juntadeandalucia.es (J.M.N.-M.) \\ 3 Department of Emergency, Hospital de la Agencia Sanitaria Alto Guadalquivir, 14550 Montilla, Spain; \\ mgutierrezs@epag.es \\ 4 Department of Investigation, Hospital Universitario Virgen de las Nieves, 18012 Granada, Spain; \\ manuela.exposito.ruiz@juntadeandalucia.es \\ * Correspondence: josegf@ugr.es
}

Received: 15 September 2020; Accepted: 30 September 2020; Published: 9 October 2020

\begin{abstract}
The increasing resistance to antibiotics is compromising the empirical treatment of infections caused by resistant bacteria. Rapid, efficient, and clinically applicable phenotypic methods are needed for their detection. This study examines the phenotypic behavior of $\beta$-lactam-resistant Gram-negative bacteria grown on ChromID ESBL medium with ertapenem, cefoxitin, and cefepime disks, reports on the coloration of colonies, and establishes a halo diameter breakpoint for the detection of carbapenemase-producing bacteria. We studied $186 \beta$-lactam-resistant Gram-negative microorganisms (77 with extended spectrum beta lactamase (ESBL), 97 with carbapenemases, and 12 with AmpC $\beta$-lactamases (AmpC)). Susceptibility profiles of Gram-negative bacteria that produced ESBL, AmpC, and carbapenemases were similar to the expected profiles, with some differences in the response to cefepime of ESBL-producing microorganisms. Coloration values did not differ from those described by the manufacturer of ChromID ESBL medium. In the screening of carbapenemase production, inhibition halo diameter breakpoints for antibiotic resistance were $18 \mathrm{~mm}$ for Enterobacterales and ertapenem, $18 \mathrm{~mm}$ for Pseudomonas and cefepime, and $16 \mathrm{~mm}$ for Acinetobacter baumannii and cefepime. This innovative phenotypic approach is highly relevant to clinical laboratories, combining susceptibility profiles with detection by coloration of high-priority resistant microorganisms such as carbapenemase-producing A. baumannii, carbapenemase-producing Pseudomonas spp., and ESBL and/or carbapenemase-producing Enterobacterales.
\end{abstract}

Keywords: beta-lactam antibiotics; resistance; Enterobacterales; Pseudomonas; Acinetobacter

\section{Introduction}

The increasing resistance to antibiotics is a major worldwide health problem, and the WHO has highlighted the threat posed by extended-spectrum $\beta$-lactamase (ESBL)-producing Enterobacteriaceae and carbapenemase-producing Acinetobacter baumannii, Pseudomonas aeruginosa, and Enterobacteriaceae [1]. Simple, rapid, effective, and inexpensive techniques are needed to screen for the presence of these pathogens in the digestive tract of infected patients and their contacts [2]. Techniques to detect ESBL-producing enterobacteria colonies include the utilization of CHROMID ESBL (bioMérieux, France), a transparent medium that contains cefpodoxime [3] and other substances 
that inhibit Gram-positive bacteria growth alongside chromogenic substrates for the presumptive identification of genera and species by their color (pink/burgundy for Escherichia coli; blue/green for Klebsiella, Enterobacter, Serratia, or Citrobacter; and light to dark brown for Proteae) [4]. The inclusion of cefoxitin (FOX), cefepime (FEP), and imipenem disks on CHROMID ESBL medium has been proposed to identify ESBL- and/or carbapenemase-producing microorganisms resistant to these antibiotics, although a halo diameter breakpoint of $15 \mathrm{~mm}$ did not prove diagnostically useful [5]. However, the diagnostic performance may be improved by considering a higher halo breakpoint $(16 \mathrm{~mm})$ and replacing imipenem with ertapenem (ERT). Carbapenemase or ESBL production is frequently studied in episodes of colonization by multi-resistant Gram-negative bacteria, and the addition of ERT, FOX, and FEP disks to this medium may offer a simple and effective method. ERT has a higher activity against ESBL-producing Enterobacterales but a lower activity against carbapenemase-producing Enterobacterales in comparison to imipenem [6]. This is why it is used after halo diameter analysis to screen for carbapenemase-producing Enterobacterales with or without ESBL. In contrast, Pseudomonas spp. and A. baumannii have a low intrinsic susceptibility to ERT [6,7], and ERT disks can be used to assess in vitro susceptibility. For its part, FEP has high antimicrobial potency against these bacteria [8] and is useful, after studying the halo diameter for Pseudomonas spp. and A. baumannii, to discriminate between isolates that produce carbapenemases, the main acquired resistance mechanism, and those that do not.

Tests that use cultures to detect colonies of resistant microorganisms offer an advantage over PCR tests because they detect viable microorganisms. Unfortunately, no commercial culture tests are available to simultaneously detect Gram-negative microorganisms with different mechanisms of resistance to $\beta$-lactam antibiotics.

With the above background, the objective of this study was to evaluate the behavior of these microorganisms, which have resistance mechanisms attributed to $\beta$-lactamases, in the ChromID ${ }^{\circledR}$ ESBL medium, using the method of antibiogram by diffusion with FOX, CEF, and ERT disks to screen for this resistance. A further aim was to determine the optimal CEF and ERT halo diameters to screen for carbapenemases, which constitute the main resistance mechanism for Pseudomonas, Acinetobacter, and Enterobacterales.

\section{Material and Methods}

\subsection{Studied Microorganisms}

This retrospective study on the growth of $\beta$-lactam-resistant Gram-negative clinical isolates included 186 strains of Gram-negative bacteria with different resistance mechanisms [9] isolated from clinical samples in the Microbiology Department of our hospital in Granada, Spain: 77 with ESBL (17 Klebsiella pneumonie, 58 E. coli and 2 Proteus mirabilis); 97 with the following carbapenemases (Table 1): New Delhi carbapenemase (NDM) (4 K. pneumoniae), Klebsiella pneumoniae carbapenemase (KPC) (2 K. pneumoniae, 1 Klebsiella oxytoca, 1 Citrobacter freundii), IMP carbapenemase (IMPase) (21 Pseudomonas spp., $1 \mathrm{~K}$. pneumoniae), Verona integron-encoded metallo-beta-lactamase (VIM) (1 C. freundii, 5 Enterobacter cloacae, 1 K. pneumoniae, 2 K. oxytoca, 2 E. coli, 7 Pseudomonas spp.), and oxacillinase (OXA) (31 A. baumannii, 14 K. pneumoniae, 2 E. cloacae, 2 E. coli, 1 C. freundii); and 12 with AmpC and FOX-resistance (4 E. coli, 2 K. pneumonie, 2 E. cloacae, 2 Enterobacter gergoviae, 1 Klebsiella aerogenes, 1 Morganella morganii). The MicroScan system (Beckman Coulter, Brea, CA, USA) and mass spectrometry (Maldi-Tof ${ }^{\circledR}$, Bruker Daltonik GmbH, Bremen, Germany) were used to identify isolates. The MicroScan microdilution system was employed to characterize resistance, followed by carbapenemase determination, when appropriate, with the Rapidec ${ }^{\circledR}$ Carba NP colorimetric test (BioMerieux, Marcy l'Etoile, France) and immunochromatography (NG5-Test Carba, NG Biotech, Guipry, France; or OXA-23 K-Set, CorisBioConcept, Gembloux, Belgium). Carbapenemase-producing type was confirmed by the Andalusian Molecular Typing Laboratory of the Spanish PIRASOA Program using mass sequencing (Illumina Inc, San Diego, CA, USA), CLC Genomics Workbench v10 software (Qiagen), and ResFinder (Lyngby, Denmark) (https://cge.cbs.dtu.dk/services/ResFinder) and CARD (Hamilton, ON, Canada) (https://card.mcmaster.ca/) databases. 
Table 1. Types of carbapenemases in Enterobacterales, Pseudomonas, and Acinetobacter, performed by the Andalusian Molecular Typing Laboratory.

\begin{tabular}{|c|c|c|c|c|c|c|c|c|c|c|c|c|c|c|}
\hline & & \multicolumn{12}{|c|}{ Types of Carbapenemases } & \multirow{3}{*}{ Total } \\
\hline & & \multicolumn{2}{|c|}{ VIM- } & \multicolumn{2}{|c|}{ KPC- } & \multirow{2}{*}{ NDM-5 - } & \multicolumn{3}{|c|}{ IMP- } & \multicolumn{4}{|c|}{ OXA } & \\
\hline & & -1 & -2 & -2 & -3 & & -8 & -16 & -23 & -48 & -23 & -58 & -245 & \\
\hline \multirow{5}{*}{ Enterobacterales } & Escherichia coli & 2 & 0 & 0 & 0 & 0 & 0 & 0 & 0 & 2 & 0 & 0 & 0 & 4 \\
\hline & Enterobacter cloacae & 5 & 0 & 0 & 0 & 0 & 0 & 0 & 0 & 2 & 0 & 0 & 0 & 7 \\
\hline & Klebsiella oxytoca & 2 & 0 & 1 & 0 & 0 & 0 & 0 & 0 & 0 & 0 & 0 & 0 & 3 \\
\hline & Klebsiella pneumoniae & 1 & 0 & 0 & 2 & 4 & 1 & 0 & 0 & 13 & 0 & 0 & 1 & 22 \\
\hline & Citrobacter freundii ${ }^{a}$ & 0 & 1 & 1 & 0 & 0 & 0 & 0 & 0 & 1 & 0 & 0 & 0 & $3^{a}$ \\
\hline \multirow{2}{*}{ Pseudomonas } & P. aeruginosa & 2 & 3 & 0 & 0 & 0 & 12 & 7 & 2 & 0 & 0 & 0 & 0 & 26 \\
\hline & P. putida & 1 & 1 & 0 & 0 & 0 & 0 & 0 & 0 & 0 & 0 & 0 & 0 & 2 \\
\hline \multirow[t]{2}{*}{ Acinetobacter } & A. baumannii & 0 & 0 & 0 & 0 & 0 & 0 & 0 & 0 & 0 & 19 & 12 & 0 & 31 \\
\hline & Total & 13 & 5 & 2 & 2 & 4 & 13 & 7 & 2 & 18 & 19 & 12 & 1 & 98 \\
\hline
\end{tabular}

a One isolate included VIM-2 and KPC-2 enzymes. 
AmpC production was defined by resistance to FOX and synergy with cloxacillin, and ESBL production was defined by resistance to cefotaxime and/or ceftazidime and synergy with clavulanic acid.

\subsection{Investigation of Bacteria Growth on CHROMID ESBL Medium}

An 0.5 McFarland suspension of each isolate was prepared from colonies grown on lamb blood agar (Becton Dikinson, Franklin Lakes, NJ, USA). Next, a sterilized swab was soaked with the homogenized suspension, excess liquid was removed, and it was uniformly seeded on one half of the plate, streaking the bacterial load on the other half with a calibrated inoculation loop. FEP (30 $\mu \mathrm{g}$, Becton Dickinson), FOX (30 $\mu$ g, Becton Dickinson), and ERT (10 $\mu$ g, Becton Dickinson) disks were then placed equidistantly on the seeded area. The medium was then incubated for $48 \mathrm{~h}$ at $37^{\circ} \mathrm{C}$, with readings at $24 \mathrm{~h}$. The isolate was considered susceptible if the inhibition halo was $\geq 1.6 \mathrm{~cm}$ at $24 \mathrm{~h}$ and resistant if it was $<1.6 \mathrm{~cm}$. We also analyzed CEF-generated inhibition halo diameters to detect carbapenemase-producing A. baumannii and Pseudomonas and ERT-generated diameters to detect carbapenemase-producing Enterobacterales. The optimal breakpoint was evaluated by constructing a receiver operating characteristic (ROC) curve based on the halo diameters.

\subsection{Statistical Analysis}

The Wilcoxon test was used to analyze the inhibition halo results for isolates with ESBL. The Kruskal-Wallis or Mann-Whitney U test was used for comparative analysis of carbapenemase enzymatic activity, comparing the inhibition halo diameters generated by the isolate. $p \leq 0.05$ was considered significant. The breakpoint of the CEF and ERT halo that best defined the presence of carbapenemase was determined according to the area under the receiver operating characteristic curve [10], after calculating the optimal breakpoints using the Youden index (sum of sensitivity and specificity minus one). IBM SPSS Statistics 19 and Microsoft Excel 2019 were used for statistical analyses.

\section{Results}

\subsection{Behavior of Susceptibility Profiles with CEF, FOX, and ERT Disks}

\subsubsection{Enterobacterales with ESBL}

Table 2 and Figure 1 display the color distribution of microorganisms. Although all isolates were resistant to FEP in microdilution, the inhibition halos varied widely (Table 3) and were almost always lower for susceptible isolates $(34 ; 44.15 \%)$ than for halos with FOX $(31 ; 91.18 \%)(p=0.00)$. Isolates were almost always $(74 ; 96.1 \%)$ susceptible to FOX (Table 3$)$, and all isolates were susceptible to ERT (halos $>20 \mathrm{~mm}$ ). Hence, almost all isolates $(96.1 \%)$ were susceptible to both ERT and FOX, and showed a variable response to FEP.

\subsubsection{Enterobacterales with AmpC}

Table 2 and Figure 1 exhibit the color distribution of the microorganisms. All isolates were susceptible to ERT and FEP (halos $>19 \mathrm{~mm}$.) and almost all were resistant to FOX (11; 91.7\%), except for one E. gergoviae isolate (halo $=17 \mathrm{~mm}$, which was resistant by microdilution (MIC $>16 \mathrm{mg} / \mathrm{L}$ ). This isolate was also resistant to amoxicillin/clavulanic acid. Hence, almost all (91.7\%) of the isolates were susceptible to ERT and FEP and resistant to FOX. 
Table 2. Distribution of isolates with ESBL according to the color of colonies on ChromID ESBL medium.

\begin{tabular}{|c|c|c|c|c|c|c|c|c|}
\hline \multirow[b]{2}{*}{ Microorganisms } & \multicolumn{5}{|c|}{ Color of Colonies on ESBL } & \multirow[b]{2}{*}{ Light Brown } & \multirow[b]{2}{*}{ Brown } & \multirow[b]{2}{*}{ Total } \\
\hline & Light Beige & Green-Blue & Light Green-Blue & Pink & Light Pink & & & \\
\hline E. coli ESBL & 1 & 0 & 0 & 54 & 3 & 0 & 0 & 58 \\
\hline E. coli AmpC & 0 & 0 & 0 & 4 & 0 & 0 & 0 & 4 \\
\hline E. coli CAR & 1 & 0 & 0 & 3 & 0 & 0 & 0 & 4 \\
\hline K. pneumoniae ESBL & 0 & 17 & 0 & 0 & 0 & 0 & 0 & 17 \\
\hline K. pneumoniae AmpC & 0 & 1 & 1 & 0 & 0 & 0 & 0 & 2 \\
\hline K. pneumoniae CAR & 0 & 22 & 0 & 0 & 0 & 0 & 0 & 22 \\
\hline P. mirabilis ESBL & 0 & 0 & 0 & 0 & 0 & 2 & 0 & 2 \\
\hline C. freundii AmpC & 1 & 0 & 0 & 0 & 0 & 0 & 0 & 1 \\
\hline C. freundii CAR & 1 & 0 & 0 & 1 & 0 & 0 & 0 & 2 \\
\hline K. aerogenes AmpC & 0 & 1 & 0 & 0 & 0 & 0 & 0 & 1 \\
\hline E. cloacae AmpC & 0 & 0 & 2 & 0 & 0 & 0 & 0 & 2 \\
\hline E. cloacae CAR & 3 & 3 & 1 & 0 & 0 & 0 & 0 & 7 \\
\hline E. gergoviae AmpC & 0 & 0 & 2 & 0 & 0 & 0 & 0 & 2 \\
\hline K. oxytoca CAR & 0 & 0 & 3 & 0 & 0 & 0 & 0 & 3 \\
\hline P. aeruginosa CAR & 14 & 0 & 0 & 0 & 0 & 0 & 12 & 26 \\
\hline P. putida CAR & 2 & 0 & 0 & 0 & 0 & 0 & 0 & 2 \\
\hline A. baumannii CAR & 31 & 0 & 0 & 0 & 0 & 0 & 0 & 31 \\
\hline Total & 54 & 44 & 9 & 62 & 3 & 2 & 12 & 186 \\
\hline
\end{tabular}

CAR: carbapenemase producer. 


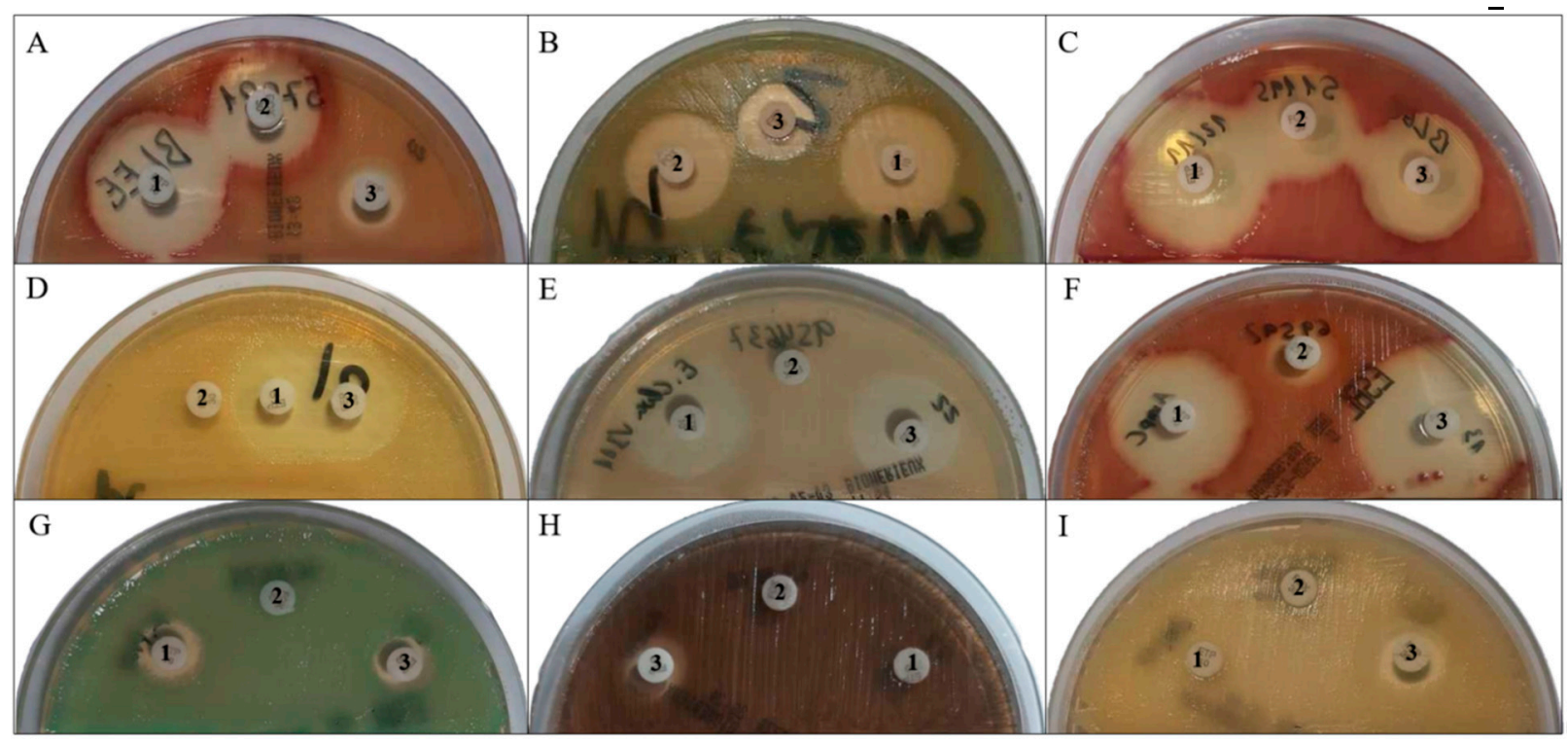

Figure 1. Coloring and susceptibility profile of the different species studied on CHROMID extended spectrum beta lactamase (ESBL) plates. (A) E. coli ESBL;(B) K. pneumoniae ESBL; (C) susceptible to cefepime E. coli ESBL; (D) E. gergoviae AmpC; (E) E. cloacae AmpC; (F) E. coli AmpC; (G) carbapenemase-producing K. pneumoniae; (H) carbapenemase-producing P. aeruginosa; (I) carbapenemase-producing A. baumannii. Antibiotic disks are marked 1 for ertapenem, 2 for cefoxitin and 3 for cefepime.

Table 3. Distribution of isolates with ESBL on ChromID ESBL medium and behavior (\%) with cefepime and cefoxitin disks.

\begin{tabular}{cccccc}
\hline & \multicolumn{2}{c}{ Cefepime } & \multicolumn{2}{c}{ Cefoxitin } & \multirow{2}{*}{ Total } \\
\cline { 1 - 5 } & Susceptible & Resistant & Susceptible & Resistant & \\
\hline E. coli & $29(37.7)$ & $29(37.7)$ & $57(74.0)$ & $1^{*}(1.3)$ & 58 \\
\hline K. pneumoniae & $3(3.9)$ & $14(18.2)$ & $15(19.5)$ & $2{ }^{* *}(2.6)$ & 17 \\
\hline P. mirabilis & $2(2.6)$ & $0(0)$ & $2(2.6)$ & $0(0)$ & 2 \\
\hline Total & $34(44.2)$ & $43(55.8)$ & $74(96.1)$ & $3(3.9)$ & 77 \\
\hline
\end{tabular}

Halos of * 14 and ${ }^{* *} 13 / 14 \mathrm{~mm}$ with MIC $=16 \mathrm{mg} / \mathrm{L}$ and susceptible to amoxicillin/clavulanic and piperacillin/ tazobactam by microdilution.

\subsubsection{Carbapenemase-producing microorganisms}

These included Enterobacterales, Pseudomonas, and A. baumannii. Table 2 and Figure 1 show the color distribution of Enterobacterales. K. oxytoca had a lighter green-blue shade in comparison to K. pneumoniae. E. cloacae had a green-blue shade of varying intensity, although some colonies were beige. C. freundii and E. coli showed a similar color pattern, with some pink and other light beige isolates, although in different percentages. Disk results were as follows: 8 (21\%) isolates were susceptible to ERT, with halos between 16 and $17 \mathrm{~mm}$ that were larger in the presence of VIM-1 and smaller in the presence of KPC-3 ( $p=0.022)$ (Figure 2); $10(26 \%)$ were susceptible to FOX, with halos between 16 and $23 \mathrm{~mm}(p=0.193)$ (Figure 3$)$; and $7(18 \%)$ were susceptible to FEP, with halos $\leq 18 \mathrm{~mm}(p=0.289)$. Hence, almost all (94.7\%) of the 36 isolates were resistant to ERT and/or FEP. 


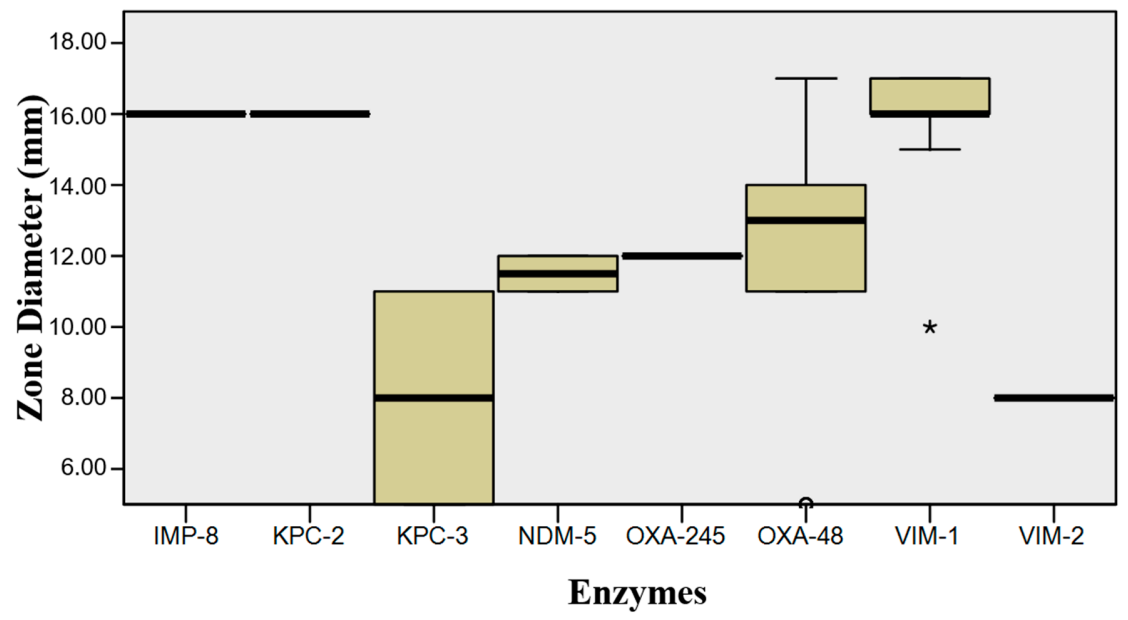

Figure 2. Distribution of diameters produced in Enterobacterales by each enzyme with ertapenem disc.

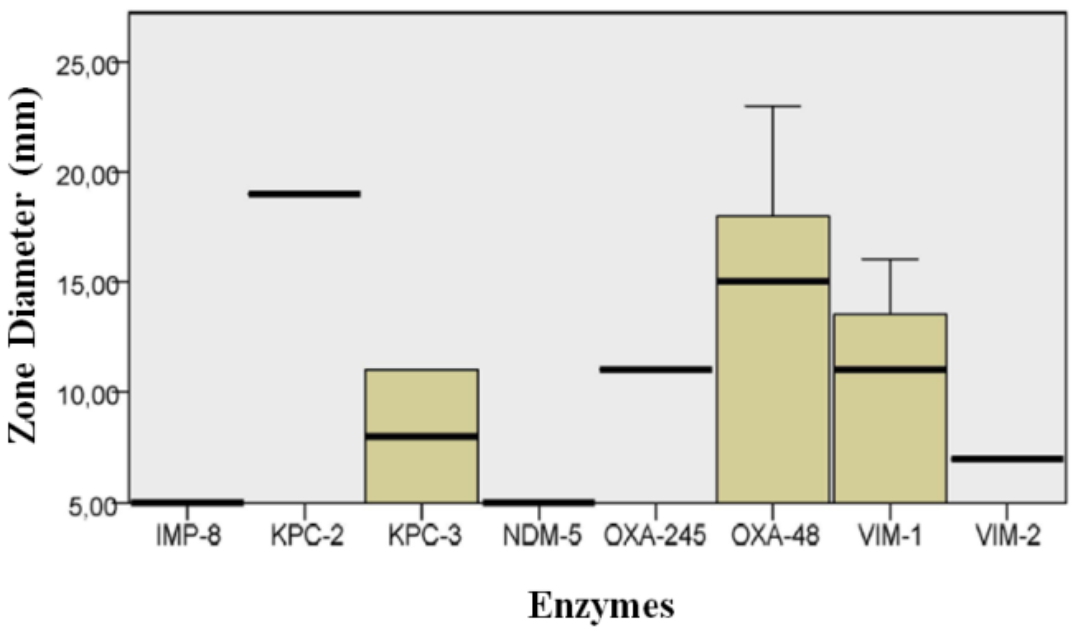

Figure 3. Distribution of diameters produced in Enterobacterales by each enzyme with cefoxitin disc.

Table 2 and Figure 1 show the color distribution of the Pseudomonas. No halos were observed with ERT or FOX for any isolate and the halo was always $<16 \mathrm{~mm}$ with FEP, being larger in the presence of VIM-2 ( $p=0.032)$.

In ChromID ESBL medium, 28 (90.3\%) of A. baumannii colonies were light beige and $3(9.7 \%)$ were beige (Table 2 and Figure 1). All isolates were resistant to all three antibiotics, although halos $<15 \mathrm{~mm}$ were generated with FEP, with no difference according to the type of OXA ( $p=$ n.s.).

\subsection{Calculation of the Breakpoint with ERT and FEP for Carbapenemase-producing Microorganisms}

\subsubsection{Enterobacterales and ERT}

We studied 115 isolates: 38 (35\%) were carbapenemase-producing, with halos between 5 and $17 \mathrm{~mm}(11.69 \pm 3.704)$ for ERT, and 77 (65\%) were ESBL-producing, with halos between 19 and $37 \mathrm{~mm}$ $(27.06 \pm 3.290)$ for ERT, as control group. The optimal breakpoint calculated using Youden's index was $18 \mathrm{~mm}$, with a sensitivity of $100 \%$ and specificity of $100 \%$ and area under the ROC curve of 1 (Figure 4 ). 


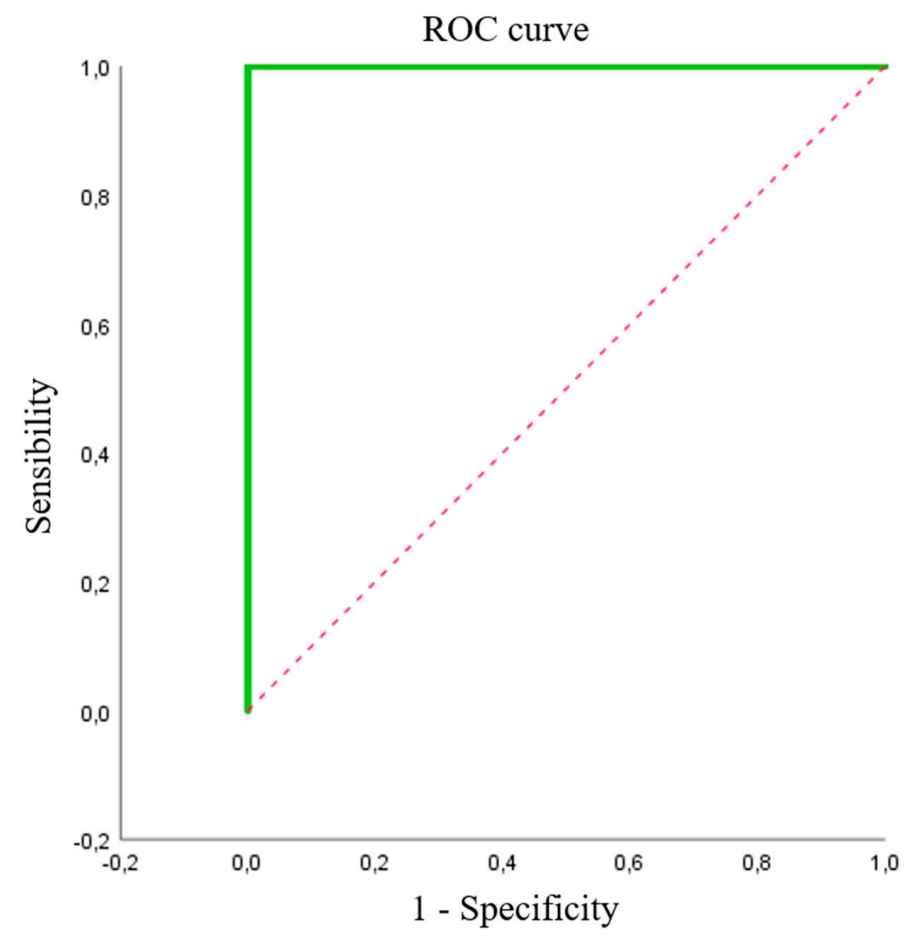

Figure 4. ROC curve calculated for Enterobacterales. The ROC curve is green and the reference diagonal line red.

\subsubsection{Pseudomonas spp. and CEF}

We studied 42 isolates: 28 (66.7\%) were carbapenemase-producing, with halos between 5 and $15 \mathrm{~mm}(10.75 \pm 3.28)$ for FEP, and $14(33.3 \%)$ were non-carbapenemase-producing P. aeruginosa, with halos between 14 and $37 \mathrm{~mm}$ for FEP $(24.43 \pm 6.63)$, as control group. The optimal breakpoint calculated using Youden's index was $18 \mathrm{~mm}$, with a sensitivity of $100 \%$ and specificity of $97.7 \%$ and area under the ROC curve of 0.977 (Figure 5).

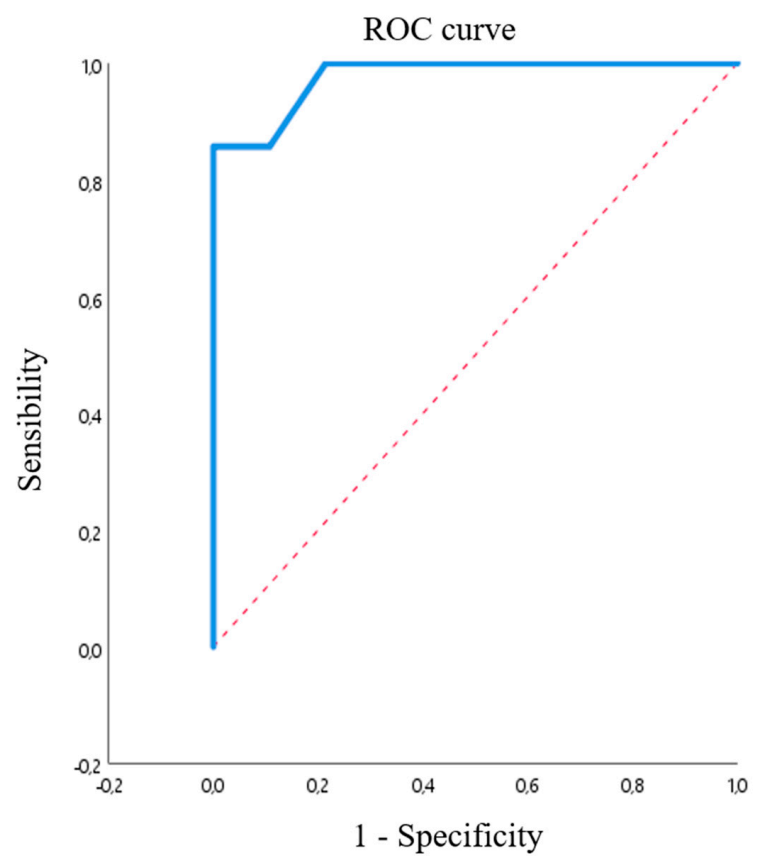

Figure 5. ROC curve calculated for P. aeruginosa. The ROC curve is blue, and the reference diagonal line is red. 


\subsubsection{A. baumannii and CEF}

We studied 71 isolates: 31 (43.7\%) were carbapenemase-producing A.baumannii, with halos between 5 and $15 \mathrm{~mm}(12.45 \pm 2.142)$ for FEP, and 40 were non-carbapenemase-producing microorganisms (2 A. baumannii, 14 P. aeruginosa, and 24 Enterobacterales), with halos between 14 and $39 \mathrm{~mm}$ for FEP (29.61 \pm 6.727$)$, as control group. The optimal breakpoint calculated using Youden's index was $16 \mathrm{~mm}$, with a sensitivity of $100 \%$ and specificity of $95 \%$ and area under the ROC curve of 0.986 (Figure 6).

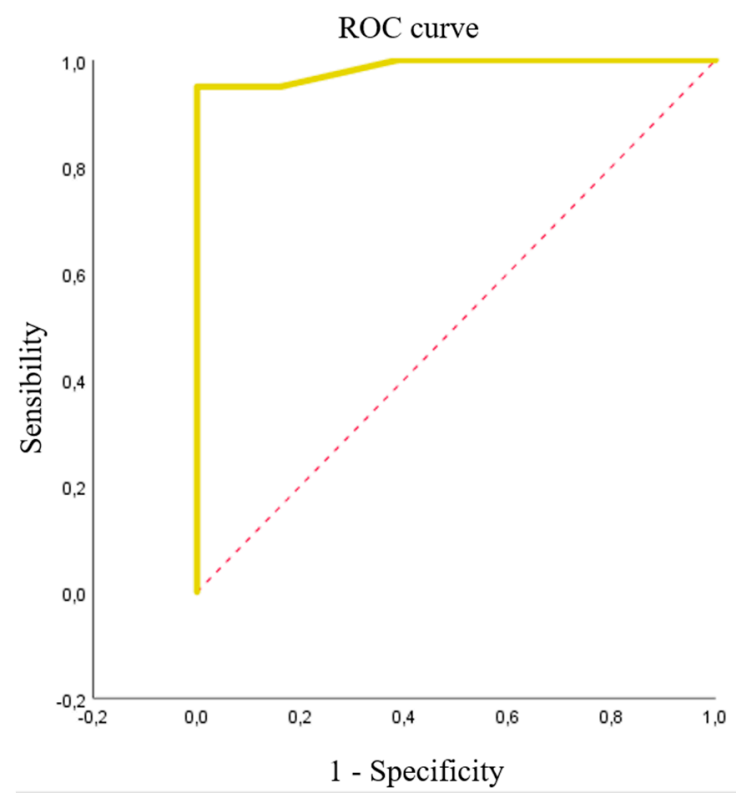

Figure 6. ROC curve calculated for A. baumannii. The ROC curve is yellow, and the reference diagonal line is red.

\section{Discussion}

\subsection{Susceptibility Profiles with CEF, FOX, and ERT Disks}

The expected susceptibility profile of ESBL-producing microorganisms shows resistance to FEP and susceptibility to FOX and ERT in antibiograms based on microdilution. All studied isolates were susceptible to ERT, reflecting the fact that carbapenems are frequently the treatment of choice against infections produced by these bacteria due to the low capacity of ESBLs to modify these molecules [11,12]. Most of the isolates were susceptible to FOX but three were resistant, which may be associated with membrane permeability reduction phenomena observed in some ESBL-producing bacteria [13], given that they were susceptible to amoxicillin/clavulanic acid and piperacillin/tazobactam. Although FOX has been proposed to treat some infections caused by ESBL-producing bacteria [14], its use must be highly controlled, because a decrease in porin number and/or expression of AmpC-type enzymes is frequently observed during the treatment $[15,16]$.

Reports on the in vitro effect of FEP against Enterobacterales are highly varied, and numerous E. coli isolates have been found to be susceptible to this antimicrobial [17]. FEP can be clinically active in some cases, although it is not recommended for infections caused by ESBL-producing bacteria because of the high failure rate [18-20].

In our series, the response to FEP of ESBL-producing isolates was also varied, with $55.8 \%$ being resistant to FEP disks when cultured in plate, contrasting with the results obtained with microdilution, when all isolates were resistant with a MIC value $>8 \mathrm{mg} / \mathrm{L}$. This discrepancy may have various explanations: 1) the lowering of the breakpoint by the CLSI in 2014 [21]; 2) reports on isolates susceptible to FEP and with ESBL from the SHV family, which are less active against this antibiotic than CTX-M-type $\beta$-lactamases [15,22]; and 3) a possible synergic effect between the FEP disk used in this 
study and the cefpodoxime in the ChromID ESBL medium, leading to the classification of numerous isolates as susceptible. The combination of both antibiotics may saturate $\beta$-lactamases, most of which have enzymatic kinetics that follow the Michaelis-Menten equation [23]. However, given that FEP and cefpodoxime will not be used in combination in the clinical setting, classification of the strains based on the microdilution method yields more relevant information. In addition, the Wilcoxon test confirmed that most isolates susceptible to FEP had a smaller halo in comparison to the FOX halos. This result is novel and can be applied in clinical diagnosis.

The expression of class C $\beta$-lactamases, such as AmpC, is constitutive and inducible in microorganisms of the Enterobacter and Citrobacter genera [24] under study here. The remaining isolates belonged to E. coli and K. pneumonia species. They do not constitutively express these types of enzyme because they lack the regulating genes, and their expression is produced by the acquisition of plasmids from the chromosomal $\beta$-lactamases of E. cloacae (ACT-1 and MIR-1), C. freundii (CMY), Hafnia alvei (ACC-1), and M. morganii (DHA-1) [25]. The observation of CMY-2 and DHA-1 in E. coli isolates and DHA-1 in K. pneumoniae isolates is very frequent [26]. The expected profile for AmpC-producing isolates is susceptibility to ERT and FEP and resistance to FOX, and the present isolates were always susceptible to ERT and FEP. The expression of AmpC-type enzymes does not usually confer resistance to carbapenems or $4^{\text {th }}$ generation cephalosporins [27] except when the overexpression of these $\beta$-lactamases is combined with a reduction in porin number [28,29], when there may be a significant increase in the MIC value of carbapenems such as ERT or imipenem. One isolate was susceptible to FOX in the ChromID ESBL medium but showed resistance in the antibiogram based on microdilution. This is unusual, because FOX is the main antibiotic used to detect this type of resistance. The diameter of the halo for this isolate was $17 \mathrm{~mm}$, suggesting the need to raise the breakpoint used to detect this type of resistance. Research in more isolates is also required.

In relation to our study of carbapenemase-producing Enterobacterales, we highlight that they included a large number of K. pneumoniae isolates (57.9\%), mainly carriers of $\beta$-lactamase OXA-48. This is attributable to the frequency of this enzyme in K. pneumoniae, which produces endemic infections in North African countries [30] and nosocomial outbreaks in Spain, among other countries [31,32]. Other E. coli and C. freundii isolates also showed this type of enzyme. Other types of $\beta$-lactamase frequently encountered in isolates of Enterobacterales include metallo- $\beta$-lactamases, such as the VIM present in E. cloacae, E. coli, K. oxytoca, and C. freundii, among other species. These isolates expressed VIM-1-type enzymes, whereas these Enterobacterales have generally been found to express VIM-2-type enzymes [33]. We also highlight the study of isolates of K. pneumoniae with NDM, which has shown a rapid expansion over the past few years [34].

There have been reports on the limited activity of OXA-48-type enzymes, which have low degradation capacity against extended-spectrum cephalosporins such as ceftazidime and carbapenems. For this reason, isolates expressing this enzyme that are identified as resistant to carbapenems frequently present other associated mechanisms, e.g., simultaneous ESBL expression and porin decrease [16,35]. The low degradation activity of OXA-48-type enzymes can lead to the clinical non-detection of its presence in some cases, thereby contributing to the spread of these isolates.

Our statistical analysis revealed that halo diameters were significantly larger for VIM-1- versus OXA-48-carrier bacteria on ERT disks, which may indicate a lower activity for this type of enzyme than for OXA-48 carbapenemases. Although the difference was not significant, the diameter range generated by bacteria expressing the KPC-3 enzyme was also lower, as in the case of the OXA-48 enzyme, although the isolated activity of KPC-3 was not sufficient to generate resistance to all $\beta$-lactams [36-38].

In the case of FOX, no significant difference was found between isolates with VIM-1 and OXA-48 enzymes, which showed a wide range of values that indicated their variable activity. OXA-48 halos had a large mean diameter, indicating the low activity of this enzyme. Once more, KPC-3-producing isolates showed small or absent halos. The reduced number of isolates expressing the remaining enzymes hampers further statistical analyses. In the case of FEP, the halo range did not significantly differ among all enzymes, observing a wide variability in KPC-3 halos and a larger diameter range for 
VIM-1 and VIM-2 enzymes. The lack of statistical significance may be attributable to the small number of isolates for each type of carbapenemase. As expected, the susceptibility profile study evidences general resistance to all three antibiotics under study; although we highlight resistance to ERT and/or FEP as a screening marker. The emergence of isolates susceptible to these antimicrobials may be explained by the finding that the isolated activity of carbapenemases in vitro is insufficient to produce a resistance profile [11,36] and indicates the need to establish a breakpoint $>16 \mathrm{~mm}$ for screening.

The carbapenemase-producing Pseudomonas were largely $P$. aeruginosa, which expressed metallo- $\beta$-lactamase-type carbapenemases, mainly type IMP. The combination of carbapenemases with other resistance mechanisms, mainly porin underexpression and AmpC overexpression [39], create high resistance to most $\beta$-lactam antibiotics, explaining the profile obtained in this study, in which $100 \%$ of isolates were resistant to ERT, FEP, and FOX. A significantly larger halo diameter was observed when VIM-2 was present, suggesting its lower activity, although these data should be interpreted with caution given the low number of isolates. No significant differences were found for the other enzyme groups. The resistance profile observed for these carbapenemase-producing Pseudomonas underscores the critical clinical importance of these microorganisms, because their presence (frequent in intensive care units) requires the use of antibiotics of last resort, e.g., colistin [40,41].

Isolates of A. baumannii only presented OXA-type class D $\beta$-lactamases, often observed in isolates of this species [35]. Their presence is increasingly implicated in the etiology of nosocomial infections due to the expression of carbapenemases, mainly OXA-23, followed by OXA-58, alongside their abundant intrinsic resistance mechanisms [41,42]. The expression of these two enzymes has previously been reported [43]. These carbapenemases and the other resistance mechanisms in A. baumannii confer a similar resistance profile to that of Pseudomonas, which showed no inhibition halos with ERT or FOX antibiotics and halos $<15 \mathrm{~mm}$ with FEP, identified as resistance by the screening test using ChromID ESBL medium. Comparative study of the halo diameters showed no significant differences between the halos produced by microorganisms expressing the two aforementioned enzymes, suggesting that the two carbapenemases exert a similar activity.

According to the manufacturer of ChromID ESBL medium, colonies of ESBL-producing E.coli are identified by their pink color, colonies of ESBL-producing Klebsiella spp., Enterobacter spp., Serratia spp., and Citrobacter spp. by their green-blue color, and those of the Proteae tribe (Proteus spp., Morganella spp. and Providencia spp.) by their dark to light brown color. Pseudomonas and Acinetobacter genera are not included in the manufacturer's list. The above colors were shown by most isolates in this study, although we highlight the white color of some isolates of E. coli (2), C. freundii (1), and E. cloacae (3). A light green-blue color was observed for K. oxytoca isolates and E. cloacae and E. aerogenes colonies, as reported by Romo-Ibáñez et al., 2020. The brown or beige color of isolates of the Pseudomonas genus is attributed by the manufacturer to the Proteae tribe, although they can be readily differentiated by the oxidase test. Most A. baumannii colonies were light beige, occasionally turning into cream beige, hampering their differentiation from some light beige Enterobacterales isolates.

Selective chromogenic media such as ChromID ESBL may facilitate work in clinical diagnosis laboratories by allowing the presumptive identification of species or groups of microorganisms, alongside their generic resistance profile, which was to cefpodoxime in the present case. If the aforementioned antibiotic disks are added to this medium, a screening strategy can be implemented to detect $\beta$-lactam resistant microorganisms.

\subsection{Breakpoint Calculation with ERT and FEP for the Screening of Carbapenemase-producing Microorganisms}

In previous studies [10], construction of a ROC curve was effective to determine a breakpoint that differentiated between isolates resistant and susceptible to certain antibiotics in media such as MacConkey agar. However, this medium does not allow the differentiation of Gram-negative microorganisms present in a clinical sample and does not inhibit the growth of susceptible isolates. Nonetheless, the MacConkey agar medium is less expensive than the ChromID ESBL medium. 
In our study, ROC curves were constructed for Enterobacterales and carbapenemase-producing non-fermenting Gram-negative bacilli in ChromID ESBL medium with ERT and FEP disks, respectively.

For carbapenemase-producing Enterobacterales, the area under the curve was 1 and sensitivity and specificity values were $100 \%$ when considering a breakpoint halo diameter of $18 \mathrm{~mm}$, likely attributable to the wide difference between the halos of non-carbapenemase-producing and carbapenemase-producing microorganisms. In other words, this test perfectly discriminated between producing and non-producing isolates, although it would be desirable to study a wider range of carbapenemases.

For Pseudomonas, the area under the ROC curve was 0.977 , the sensitivity was $100 \%$, and the specificity was $85.7 \%$ for a breakpoint of $18 \mathrm{~mm}$. As above, these data should be interpreted with caution due to the reduced number of isolates.

For A. baumannii, a different bacterial pool had to be used to study halos produced with FEP, because of the difficulty in finding $A$. baumannii susceptible to $\beta$-lactams in our setting. The area under the ROC curve was 0.986 for a breakpoint halo diameter of $16 \mathrm{~min}$, which yielded sensitivity of $100 \%$ and specificity of $95 \%$. In other words, as with the breakpoint for Pseudomonas, there is a high likelihood of correctly evaluating isolates as resistant when the halo is below the breakpoint and as susceptible when the halo is equal to or larger than the breakpoint diameter. Besides the small number of resistant isolates, a further limitation of the test was the use of bacteria other than A. baumannii as negative control group.

\subsection{Study Limitations for Its Application in Clinical Practice}

The combined presence in a clinical isolate of different resistance mechanisms (porin decrease, $\beta$-lactamases, ... ) generates different antibiotic susceptibility profiles for each microorganism, and the presence of carbapenemases needs to be detected by using multiple phenotypic tests, such as a combination of antibiogram screening by diffusion, placing disks on ChromID ESBL medium, with an immunochromatography test. However, molecular techniques are required to confirm the expression of carbapenemases and identify resistance genes, given the clinical relevance of this information in hospitals. Further studies are needed in a larger number of isolates to confirm these preliminary results and the value of the ESBL medium with disks for screening clinical samples with ESBL- and Carbapenemase-mediated resistances.

\section{Conclusions}

Microbiology laboratories should provide economic and easily-applied screening tests to detect microorganisms resistant to $\beta$-lactams, given their increasing clinical importance. This study highlights the usefulness of ChromID ESBL medium with the placement of antibiotic disks to screen for resistant Gram-negative microorganisms when inhibition haloes are $<16 \mathrm{~mm}$. In this way, ESBL-producing Enterobacterales are susceptible to ERT and FOX disks, and AmpC producers are resistant to FOX and susceptible to ERT and FEP. Most carbapenemase producers are resistant to ERT and/or FEP, although a better diagnostic performance was achieved with ERT and a halo breakpoint of $18 \mathrm{~mm}$. Carbapenemase-producing A. baumannii and Pseudomonas spp. are resistant to ERT, FOX, and FEP, although an excellent diagnostic performance was obtained for Pseudomonas spp. with FEP and a halo breakpoint of $18 \mathrm{~mm}$. Alongside the differential coloration of colonies, this approach permits the implementation of a simple and fast method to screen for resistant microorganisms in clinical microbiology laboratories, allowing focus on the search for a specific resistance mechanism in detected microorganisms.

Author Contributions: Conceptualization, J.G.-F. and J.M.N.-M.; methodology, J.G.-F. and F.M.-R.; software, M.E.-R.; validation, J.G.-F., F.M.-R., E.C.-H. and M.G.-S.; formal analysis, J.G.-F. and F.M.-R.; investigation, F.M.-R. and E.C-H.; writing-original draft preparation, F.M.-R.; writing-review and editing, F.M.-R. and J.G.-F.; visualization, M.G.-S.; supervision, J.G.-F. All authors have read and agreed to the published version of the manuscript. 
Funding: This research received no external funding.

Acknowledgments: The authors are grateful to the Andalusian Molecular Typing Laboratory of the Spanish PIRASOA Program for the genetic studies of the clinical isolates.

Conflicts of Interest: The authors have no conflict of interest to declare.

\section{References}

1. WHO. WHO Publishes List of Bacteria for Which New Antibiotics are Urgently Needed. Available online: https://www.who.int/news-room/detail/27-02-2017-who-publishes-list-of-bacteria-for-which-newantibiotics-are-urgently-needed (accessed on 14 August 2020).

2. Oteo, J.; Bou, G.; Chaves, F.; Oliver, A. Microbiological methods for surveillance of carrier status of multiresistant bacteria. Enferm. Infec. Microbiol. Clin. 2017, 35, 667-675. [CrossRef] [PubMed]

3. Paniagua, R.; Valverde, A.; Coque, T.M.; Baquero, F.; Cantón, R. Assessment of prevalence and changing epidemiology of extended- spectrum $\beta$-lactamase-producing Enterobacteriaceae fecal carriers using a chromogenic medium. Diagn. Microbiol. Infect. Dis. 2010, 67, 376-379. [CrossRef] [PubMed]

4. Del Castillo, M.C.; López-Cerezo, L.; Casal, M.; Pascual, A. Evaluation of chromID ESBL medium for detecting carriers of extended-spectrum beta-lactamase-producing Enterobacteriaceae. Enferm. Infecc. Microbiol. Clin. 2011, 9, 471-472. [CrossRef] [PubMed]

5. Romo-Ibáñez, Á.; Calatrava-Hernández, E.; Gutiérrez-Soto, B.; Pérez-Ruiz, M.; Navarro-Marí, J.M.; Gutiérrez-Fernández, J. High clinical impact of rapid susceptibility testing on CHROMID ESBL ${ }^{\circledR}$ medium directly from swabs. Ann. Trans. Med. 2020, 8, e604. [CrossRef] [PubMed]

6. Fresnadillo Martínez, M.J.; García García, M.I.; García Sánchez, E.; García Sánchez, J.E. Available carbapenems: Properties and differences. Enferm. Infecc. Microbiol. Clin. 2010, 28 (Suppl. 2), 53-64.

7. Araújo, M.; Santos, C.M.; Lages, D. Carbapenem resistant Enterobacteriaceae-the basics for every medical specialty. Port. J. Nephrol. Hyp. 2019, 33, 176-181. [CrossRef]

8. Peleg, A.Y.; Seifert, H.; Paterson, D.L. Acinetobacter baumannii: Emergence of a successful pathogen. Clin. Microbiol. Rev. 2008, 21, 538-582. [CrossRef]

9. CLSI (Clínical and Laboratory Standards Institute). M100. In Performance Standards for Antimicrobial Susceptibility Testing, 30th ed.; CLSI: Annapolis Junction, MD, USA, 2020.

10. Workneh, M.; Wang, R.; Kazmi, A.Q.; Chambers, K.K.; Opene, B.N.A.; Lewis, S.; Goodman, K.; Tamma, P.D.; Carroll, K.C.; Milstone, A.M.; et al. Evaluation of the direct macconkey method for identification of carbapenem-resistant gram-negative organisms from rectal swabs: Re-evaluating zone diameter cutoffs. J. Clin. Microbiol. 2019, 57, 12. [CrossRef] [PubMed]

11. Pitout, J.D.D. Infections with extended-spectrum $\beta$-lactamase-producing Enterobacteriaceae. Drugs 2010, 70, 313-333. [CrossRef]

12. Vardakas, K.Z.; Tansarli, G.S.; Rafailidis, P.I.; Falagas, M.E. Carbapenems versus alternative antibiotics for the treatment of bacteraemia due to enterobacteriaceae producing extended-spectrum $\beta$-lactamases: A systematic review and meta-analysis. J. Antimicrob. Chemother. 2012, 67, 2793-2803. [CrossRef]

13. Pangon, B. In-vivo selection of a cephamycin-resistant, porin-deficient mutant of Klebsiella pneumoniae producing a TEM-3 $\beta$-lactamase. J. Infect. Dis. 1989, 159, 1005-1007. [CrossRef] [PubMed]

14. Demonchy, E.; Courjon, J.; Ughetto, E.; Durand, M.; Risso, K.; Garraffo, R.; Roger, P.M. Cefoxitin-based antibiotic therapy for extended-spectrum $\beta$-lactamase-producing Enterobacteriaceae prostatitis: A prospective pilot study. Int. J. Antimicrob. Agents 2018, 51, 836-841. [CrossRef] [PubMed]

15. Rupp, M.; Fey, P.D.; Rupp, M.E.; Fey, P.D. Extended spectrum $\beta$-lactamase (ESBL)-producing Enterobacteriaceae considerations for diagnosis, prevention and drug treatment. Drugs 2003, 63, 353-365. [CrossRef] [PubMed]

16. Sorlozano, A.; Salmeron, A.; Martinez-Checa, F.; Villegas, E.; Luna, J.D.; Gutierrez, J. Low impact of OmpC and OmpF on susceptibility to antibiotics in clinical isolates of Escherichia coli producers of extended-spectrum beta-lactamases. In Antibiotic Resistance: Causes and Risk Factors, Mechanisms and Alternatives; Bonilla, A.R., Munizeds, K.P., Eds.; Nova Biomedical Books: New York, NY, USA, 2009; pp. 261-270. ISBN 978-1-60741-623-4. 
17. Flament-Simon, S.C.; Nicolas-Chanoine, M.H.; García, V.; Duprilot, M.; Mayer, N.; Alonso, M.P.; García-Meniño, I.; Blanco, J.E.; Blanco, M.; Blanco, J. Clonal structure, virulence factor-encoding genes and antibiotic resistance of Escherichia coli, causing urinary tract infections and other extraintestinal infections in humans in spain and france during 2016. Antibiotics 2020, 9, 161. [CrossRef] [PubMed]

18. Dangelo, R.G.; Johnson, J.K.; Bork, J.T.; Heil, E.L. Treatment options for extended-spectrum beta-lactamase (ESBL) and AmpC-producing bacteria. Expert Opin. Pharmacoth. 2016, 17, 953-967. [CrossRef]

19. Jiménez-Guerra, G.; Heras-Cañas, V.; Béjar Molina, L.D.C.; Sorlózano-Puerto, A.; Navarro-Marí, J.M.; Gutiérrez-Fernández, J. Extended-spectrum beta-lactamase-producing Escherichia coli and Klebsiella pneumoniae from urinary tract infections: Evolution of antimicrobial resistance and treatment options. Med. Clin. (Barc.) 2018, 150, 262-265. [CrossRef]

20. Paterson, D.L.; Ko, W.; Von Gottberg, A.; Casellas, M.; Mulazimoglu, L.; Klugman, K.P.; Bonomo, R.A.; Rice, L.B.; Mccormack, J.G.; Yu, V.L.; et al. Outcome of cephalosporin treatment for serious infections due to apparently susceptible organisms producing extended-spectrum beta-lactamases. J. Clin. Microbiol. 2001, 39, 2206. [CrossRef]

21. Patel, H.B.; Lusk, K.A.; Cota, J.M. The role of cefepime in the treatment of extended-spectrum beta-lactamase infections. J. Pharm. Pract. 2019, 32, 458-463. [CrossRef]

22. Tzouvelekis, L.S.; Tzelepi, E.; Tassios, P.T.; Legakis, N.J. CTX-M-type $\beta$-lactamases: An emerging group of extended-spectrum enzymes. Int. J. Antimicrob. Agents 2000, 14, 137-142. [CrossRef]

23. Livermore, D.M. Beta-lactamases in laboratory and clinical resistance. Clin Microbiol Rev. 1995, 8, 557-584. [CrossRef]

24. Jacoby, G.A. AmpC B-Lactamases. Clin. Microbiol. Rev. 2009, 22, 161-182. [CrossRef]

25. Bush, K.; Bradford, P.A. Epidemiology of $\beta$-Lactamase-Producing Pathogens. Am. Soc. Microbiol. 2020, 33, 1-37. [CrossRef] [PubMed]

26. Miró, E.; Agüero, J.; Larrosa, M.N.; Fernández, A.; Conejo, M.C.; Bou, G.; González-López, J.J.; Lara, N.; Martínez-Martínez, L.; Oliver, A.; et al. Prevalence and molecular epidemiology of acquired AmpC $\beta$-lactamases and carbapenemases in Enterobacteriaceae isolates from 35 hospitals in Spain. Eur. J. Clin. Microbiol. Infect. Dis. 2012, 32, 253-259. [CrossRef] [PubMed]

27. Jiménez-Guerra, G.; Borrego-Jiménez, J.; Gutiérrez-Soto, B.; Expósito-Ruiz, M.; Navarro-Marí, J.M.; Gutiérrez-Fernández, J. Susceptibility evolution to antibiotics of Enterobacter cloacae, Morganella morganii, Klebsiella aerogenes and Citrobacter freundii involved in urinary tract infections: An 11-year epidemiological surveillance study. Enferm. Infecc. Microbiol. Clin. 2020, 38, 166-169. [CrossRef] [PubMed]

28. Shin, S.Y.; Bae, I.K.; Kim, J.; Jeong, S.H.; Yong, D.; Kim, J.M.; Lee, K. Resistance to carbapenems in sequence type 11 Klebsiella pneumoniae is related to DHA-1 and loss of OmpK35 and/or OmpK36. J. Med. Microbiol. 2012, 61, 239-245. [CrossRef]

29. Tsai, Y.K.; Liou, C.H.; Fung, C.P.; Lin, J.C.; Siu, L.K. Single or in combination antimicrobial resistance mechanisms of Klebsiella pneumoniae contribute to varied susceptibility to different carbapenems. PLOS ONE 2013, 8, e79640. [CrossRef]

30. Mairi, A.; Pantel, A.; Sotto, A.; Lavigne, J.P.; Touati, A. OXA-48-like carbapenemases producing Enterobacteriaceae in different niches. Eur. J. Clin. Microbiol. Infect. Dis. 2018, 37, 587-604. [CrossRef]

31. López-Camacho, E.; Paño-Pardo, J.R.; Ruiz-Carrascoso, G.; Wesselink, J.J.; Lusa-Bernal, S.; Ramos-Ruiz, R.; Ovalle, S.; Gómez-Gil, R.; Pérez-Blanco, V.; Pérez-Vázquez, M.; et al. Population structure of OXA-48producing Klebsiella pneumoniae ST405 isolates during a hospital outbreak characterised by genomic typing. J. Glob. Antimicrob. Resist. 2018, 15, 48-54. [CrossRef]

32. Robustillo-Rodela, A.; Pérez-Blanco, V.; Espinel Ruiz, M.A.; Ruiz Carrascoso, G.; Figueira Iglesias, J.C.; Abad Martín, D. Successful control of 2 simultaneous outbreaks of OXA-48 carbapenemase-producing Enterobacteriaceae and multidrug-resistant Acinetobacter baumannii in an intensive care unit. Am. J. Infect. Control 2017, 45, 1356-1362. [CrossRef]

33. Ayoub Moubareck, C.; Halat, D.H. The current burden of carbapenemases: Review of significant properties and dissemination among. Antibiotics 2020, 9, 186-220.

34. Wu, W. NDM metallo-beta-lactamases and their bacterial producers in health care settings. Clin. Microbiol. Rev. 2019, 32, 115-118. [CrossRef] [PubMed]

35. Pitout, J.D.D.; Peirano, G.; Kock, M.M.; Strydom, K.A.; Matsumura, Y. The global ascendency of OXA-48-type carbapenemases. Clin. Microbiol. Rev. 2020, 33, 1-48. [CrossRef] [PubMed] 
36. Walther-Rasmussen, J.; Høiby, N. Class A carbapenemases. J. Antimicrob. Chemother. 2007, 60, 470-482. [CrossRef] [PubMed]

37. Soria-Segarra, C.; Soria-Segarra, C.; Catagua-González, A.; Gutiérrez-Fernández, J. Carbapenemase producing Enterobacteriaceae in intensive care units in Ecuador: Results from a multicenter study. J. Infect. Public Health 2020, 13, 80-88. [CrossRef]

38. Soria-Segarra, C.; González-Bustos, P.; López-Cerero, L.; Fernández-Cuenca, F.; Rojo-Martín, M.D.; Fernández-Sierra, M.A.; Gutiérrez-Fernández, J. Tracking KPC-3-producing ST-258 Klebsiella pneumoniae outbreak in a third-level hospital in Granada (Andalusia, Spain) by risk factors and molecular characteristics. Mol. Biol. Rep. 2020, 47, 1089-1097. [CrossRef]

39. Dolejska, M.; Papagiannitsis, C.C. Plasmid-mediated resistance is going wild. Plasmid. 2018, 99, 99-111. [CrossRef]

40. Pachori, P.; Gothalwal, R.; Gandhi, P. Emergence of antibiotic resistance Pseudomonas aeruginosa in intensive care unit; a critical review. Genes Dis. 2019, 6, 109-119. [CrossRef]

41. Jiménez-Guerra, G.; Heras-Cañas, V.; Gutiérrez-Soto, M.; Navarro-Marí, J.M.; Gutiérrez-Fernández, J. Urinary tract infection by Acinetobacter baumannii and Pseudomonas aeruginosa: Evolution of antimicrobial resistance and therapeutic alternatives. J. Med. Microbiol. 2018, 67, 790-797. [CrossRef]

42. Lupo, A.; Haenni, M.; Madec, J.-Y. Antimicrobial Resistance in Acinetobacter spp. and Pseudomonas spp. Microbiol. Spectrum 2018, 6, 1-16.

43. Sawa, T.; Kooguchi, K.; Moriyama, K. Molecular diversity of extended-spectrum $\beta$-lactamases and carbapenemases, and antimicrobial resistance. J. Intensive Care 2020, 8, 13. [CrossRef]

(C) 2020 by the authors. Licensee MDPI, Basel, Switzerland. This article is an open access article distributed under the terms and conditions of the Creative Commons Attribution (CC BY) license (http://creativecommons.org/licenses/by/4.0/). 